ISSN 0001-6002/99/41/4/6-15

Acta Médica Costarricense, (C1999

Revisión

Colegio de Médicos y Cirujanos

\title{
La Tecnología en el Laboratorio Clínico del Futuro
}

\author{
Marcela Truque Arias *
}

\begin{abstract}
Resumen: Se discuten algunas de las tecnologías que tendrán un gran impacto en la práctica del laboratorio clínico en el futuro. Estas son: el diagnóstico molecular, las pruebas rápidas que se hacen "al lado del paciente", el análisis de imágenes, la robótica y los sistemas manejo de la información. Se presenta una revisión de las principales aplicaciones y ventajas que tendrán estos nuevos avances tecnológicos en el desempeño de los laboratorios clínicos en los próximos años.
\end{abstract}

Descriptores: tecnología, diagnóstico molecular, pruebas rápidas, biosensores, robótica, análisis de imágenes, manejo de información, telepatología, laboratorio clínico.

\section{Introducción}

Vivimos en un mundo cambiante inmerso en un proceso de globalización. Las telecomunicaciones, los sistemas de transporte y las tecnologías emergentes están trasformando nuestro planeta. Como países en desarrollo, vivimos crisis multidimensionales que incluyen cambios sociales, económicos, ecológicos, políticos y tecnológicos. Estas transformaciones, como es de esperar, afectan el campo de la salud.

El cambio es inevitable en prácticamente todos los campos del quehacer humano, pero sobre todo en el laboratorio clínico. El laboratorio está en un proceso de transformación en el cual la tecnología está asumiendo un papel primordial. Al acercarnos al siglo XXI, los avances en la automatización y en la informática nos obligan a implantar nueva tecnología. El laboratorio, como todos los demás servicios de salud, debe adaptarse a estos cambios. ${ }^{1}$

Los esfuerzos para reformar el sector salud están obligando a los laboratorios clínicos a aumentar su eficiencia y a reducir costos y tiempos de respuesta. Se está viendo como la adopción de nuevas tecnologías es un mecanismo para poder cumplir con estos objetivos..$^{2,3}$

Abreviaturas: PCR, Reacción en cadena de la polimerasa; ADN, Ácido desoxirribonucleico; HIV, Virus de la inmunodeficiencia humana; HLA, Antígenos de histocompatibilidad.

* Laboratorio de Análisis Clínicos y Banco de Sangre "San José".

Correspondencia: Marcela Truque Arias, Apartado postal 4557-1000, San José, Costa Rica.

6 AMC, diciembre 1999, vol 41 (4)
Cinco tecnologías tendrán un gran impacto en la práctica del laboratorio clínico en los próximos años. Estas son el diagnóstico molecular, las pruebas rápidas que se hacen "al lado del paciente", el análisis de imágenes, la robótica y los sistemas de manejo de la información.

Con la forma tan rápida con que ocurren nuevos avances, se puede asumir que continuarán ocurriendo cambios dramáticos en la tecnología. En consecuencia, cada vez será más importante para los profesionales médicos y de laboratorio estar al tanto de nuevos descubrimientos, para poder comprenderlos y anticipar la forma en que deberán ser asimilados e integrados al sistema de salud. ${ }^{4}$

\section{Diagnóstico molecular}

El progreso que ha sufrido la biología molecular ha sido revolucionario, a partir de la introducción, a mediados de los ochenta, de la herramienta con mayor aplicación en el laboratorio clínico: la reacción en cadena de la polimerasa, más conocida por PCR. ${ }^{5}$

La reacción en cadena de la polimerasa es una forma simple y, especialmente, muy rápida de multiplicar el ADN (ácido desoxirribonucleico) presente en diferentes muestras biológicas, para posibilitar su identificación, al permitir la obtención de millones de copias de una determinada secuencia de nucleótidos. ${ }^{6}$

La reacción de la polimerasa (PCR) se utiliza con el fin de amplificar un segmento de ADN, a través de una serie de ciclos repetitivos consistentes en tres pasos. El primero es la desnaturalización por calor del ADN de la bacteria o virus o de alguna célula humana o no. Luego de desnaturalizado el ADN, se 
realiza la separación física de las dos cadenas, mediante la incubación de la muestra con alta temperatura $\left(93-97^{\circ} \mathrm{C}\right)$. Estas permanecerán separadas libres en la solución hasta que la reacción sea enfriada $\left(35-40^{\circ} \mathrm{C}\right)$ para permitir que los "primers" o "sondas", que son secuencias específicas complementarias, se unan a las secuencias buscadas y se realice la hibridización. El tercer paso consiste en la polimerización, elongación o extensión del complejo formado por las sondas y el ADN, mediante cambios cíclicos de temperatura repetidos un gran número de veces y por la acción de una enzima llamada "Taq polimerasa" la cual es muy termoestable y es la que, en buena cuenta, ha permitido la automatización del proceso de PCR en una forma relativamente sencilla. $^{6}$

Las aplicaciones diagnósticas de las pruebas moleculares incluyen campos tan diversos como enfermedades infecciosas, enfermedades genéticas, oncología y medicina legal. Las pruebas moleculares han dejado de ser exóticas, distantes y complejas y están llegando a todos los laboratorios y revolucionando el diagnóstico rápido de organismos patogénicos. ${ }^{7}$

La lista de pruebas moleculares con utilidad clínica potencial es cada día más extensa; las aplicaciones de estas novedosas técnicas parecen no tener límites. Algunas de éstas, como las pruebas para el virus de la inmunodeficiencia humana $(\mathrm{VIH})$, hepatitis $\mathrm{C}$, tuberculosis, clamidia, papilomavirus humano, herpes y micoplasma, ya se encuentran disponibles en kits comerciales. En el campo de las enfermedades infecciosas la metodología diagnóstica de la PCR ha dado sorprendentes resultados, principalmente por tratarse de un método extremadamente sensible y específico para el diagnóstico definitivo de diferentes agentes patógenos.

Los métodos moleculares se están utilizando para realizar estudios epidemiológicos de enfermedades infecciosas. Por ejemplo, las investigaciones de brotes nosocomiales de infecciones fúngicas se han facilitado enormemente por la prueba de PCR. Es posible que, en el futuro, estos métodos moleculares van a suplementar e incluso a reemplazar los métodos tradicionales para detección de Candida albicans en septicemias, aspergilosis invasivas y neumonías por Pneumocystis carinii. ${ }^{8}$

La técnica de PCR se está empleando en el estudio de la carga viral en infecciones por el VIH. Con esta prueba se puede determinar la cantidad de ARN viral circulante en los pacientes infectados ayudando en predecir la progresión de la enfermedad y a establecer la respuesta a la intervención terapéutica.

Las técnicas moleculares pueden reducir enormemente el tiempo de respuesta para la identificación de algunos microorganismos del laboratorio clínico. Por ejemplo, la confirmación de bacilos ácido resistentes, como Mycobacteriun tuberculosis o Mycobacteriun avium-intracelulare mediante cultivo, puede hacerse con pruebas moleculares en pocas horas después de aislar el microorganismo. Los métodos convencionales de identificación pueden requerir más de 2 a 4 semanas. De la misma forma, la identificación de hongos como Histoplasma capsulatum con técnicas moleculares puede hacerse en menos de 24 horas, con un mínimo de crecimiento, mientras que la identificación tradicional toma 1 a 2 semanas o más. ${ }^{9}$

Las pruebas de $\mathrm{ADN}$ en oncología, como las de detección de los rearreglos en los genes de las células T ó B y la detección de oncogenes como la translocación $\mathrm{t}^{9: 22}$ del cromosoma Philadelphia, han probado ser útiles en la detección de pequeñas cantidades de células tumorales en leucemias y linfomas y se ha demostrado su utilidad clínica en muchas otras circunstancias. ${ }^{10}$

La posibilidad de medir marcadores tumorales a nivel molecular, le da otra dimensión al laboratorio clínico. La habilidad de determinar cantidades ínfimas de moléculas biológicas le presenta al laboratorio clínico la oportunidad de contribuir en la medicina preventiva y detección temprana de enfermedades. ${ }^{11}$

También, esta tecnología tiene aplicaciones diagnósticas en el estudio de enfermedades genéticas como la distrofia muscular de Duchenne y la fibrosis quística. Las pruebas para enfermedades monogénicas, como la anemia drepanocítica, han demostrado ser útiles para el manejo prenatal de madres en riesgo. ${ }^{12}$

Las aplicaciones potenciales para las pruebas genéticas son inmensas. La mayoría de las enfermedades están influenciadas o causadas por cambios en el genoma del paciente. Con el tiempo, la nueva información genética se transformará en pruebas clínicas para la predicción del riesgo de futuras enfermedades. Muchas de las pruebas genéticas serán altamente automatizadas. ${ }^{12}$

Actualmente, se dispone de pruebas de tamizaje para la detección de predisposición genética a ciertas formas de cáncer de colon y mama y a la enfermedad de Alzheimer. Estos ejemplos demuestran el potencial a gran escala que tienen estas pruebas para el tamizaje de poblaciones en riesgo.

También se ha introducido el uso de la técnica de PCR para el estudio del complejo mayor de histocompatibilidad (antígenos HLA) para transplante de médula ósea, estudios de paternidad e identificación de tejidos.

Para los laboratorios clínicos y de patología, la PCR será la herramienta de elección para el próximo siglo, conforme se van desarrollando y refinando nuevas aplicaciones. La PCR cambiará la práctica de la medicina porque juega papeles en todos los aspectos de la atención del paciente desde el diagnóstico hasta el seguimiento del tratamiento..$^{13}$

El costo inherente de las pruebas de diagnóstico molecular es de importancia significativa para cualquier organización. El valor clínico y el costo potencial de estas pruebas se debe evaluar detenidamente, antes de introducir la tecnología en el laboratorio. Entre las ventajas de la tecnología de diagnóstico molecular que 
se mencionan están: la disminución de los períodos de hospitalización resultado de un diagnóstico temprano y definitivo; reducción en el uso de exámenes no definitivos, la reducción en servicios de diagnóstico auxiliar como radiología, ultrasonido y endoscopías, mayor posibilidad de predecir la respuesta a varias terapias costosas y el curso clínico de la enfermedad. ${ }^{14}$

Los médicos y los profesionales de laboratorio deben interesarse en las pruebas moleculares para poder seguir el desarrollo de la medicina moderna y poder informar a sus pacientes y al público en general las nuevas posibilidades y limitaciones, así como los riesgos de las pruebas moleculares.

\section{Pruebas rápidas}

Los constantes avances en la miniaturización y en la tecnología de biosensores permitirán que cada vez sean más los analitos que se puedan estudiar "al lado del paciente".

Conforme se perfeccionan los cuidados intensivos y los transplantes, aumenta la necesidad de utilizar pruebas que brinden resultados más rápidos. ${ }^{15}$ Una prueba de laboratorio rápida, en la evaluación de una emergencia, como lo sería un paciente con un dolor en el pecho, podría contribuir a estratificar el riesgo del paciente de manera que se pueda elegir rápidamente la opción terapéutica más adecuada. ${ }^{16,17}$ Poder analizar rápidamente ciertas pruebas de laboratorio seleccionadas, es esencial para el manejo de pacientes en estado crítico. ${ }^{18,19}$

Los biosensores son herramientas analíticas capaces de proveer resultados cualitativos y cuantitativos. Consisten en sistemas de bio-reconocimiento, típicamente enzimas o proteínas ligadoras, como los anticuerpos, que son inmovilizados sobre una superficie de transductores físicoquímicos. El término inmunosensor se utiliza frecuentemente para describir biosensores que utilizan anticuerpos como su sistema de bio-reconocimiento. Los sistemas de bio-reconocimiento puede incluir además de enzimas y ácidos nucleicos, bacterias y células e incluso tejidos de organismos superiores. Las interacciones específicas que se forman entre el analito y esta capa complementaria, producen un cambio físicoquímico que se puede detectar y medir por medio de un transductor. ${ }^{20}$

Los transductores pueden ser de varias clases dependiendo de los parámetros que se midan (potenciométricos, amperométricos, ópticos y otros físicoquímicos). Los transductores potenciométricos miden la carga en la superficie de un electrodo. Los sensores amperométricos miden corrientes generadas cuando los electrones se intercambian entre un sistema biológico y un electrodo. Los ópticos correlacionan los cambios en concentración, masa o en las características de la luz. Otros sensores fisicoquímicos miden las interacciones biológicas a través de cambios en entalpía, conducción iónica y masa. ${ }^{20}$

En el campo médico, los biosensores están permitiendo que se puedan realizar análisis clínicos en los salones de pacientes y en las unidades de emergencias y cuidados intensivos, en vez de que se hagan en laboratorios centralizados. La rapidez de estas pruebas se debe principalmente a que no requieren la adición de reactivos ni necesitan de fases de separación o lavados..$^{20,21}$ Entre las ventajas de estos analizadores de sangre total se encuentran: el tiempo reducido de respuesta, el que no se necesita centrifugación, que se conserva el volumen sanguíneo y hay mayor exactitud. ${ }^{16}$

La implementación exitosa de la tecnología de sensores de fase sólida promete simplificar los procedimientos de inmunoensayos, como lo hicieron los autoanalizadores hace más de 20 años para el perfil de metabolitos sanguíneos. Es muy posible que con el advenimiento de biosensores automatizados y rentables, se puedan realizar cada vez más pruebas "al lado del paciente". ${ }^{22}$

Entre los biosensores que han tenido el mayor éxito hasta la fecha se encuentran los dispositivos para control de la glucosa. Los biosensores de este tipo son electrónicos y dependen de enzimas que reconocen y catalizan reacciones de glucosa con la generación de especies redox que son detectadas electroquímicamente.

En la actualidad muchas instituciones, en otros países, realizan pruebas de glucosa, hemoglobina, electrólitos y gases arteriales, "al pie de la cama del paciente". Esta estrategia tiene la ventaja de que reduce mucho los costos de los exámenes comparado con el análisis en el laboratorio central; además, se evitan las demoras asociadas con el envío y espera de los resultados. ${ }^{23.24}$

Algunos investigadores han demostrado que la determinación rápida de los gases arteriales reduce la estadía promedio de los pacientes en cuidados intensivos. Por ejemplo, en el caso de las unidades postoperatorias torácica y cardiovascular, estos pacientes requieren frecuentemente los resultados de los gases arteriales, que se realizan en el laboratorio, para poder ajustar los ventiladores respiratorios. Se ha calculado que si se realizaran estas pruebas "al lado del paciente" se reduce el número promedio de horas en un ventilador en más de 15 horas, de $53 \%$ a menos de $31 \%$, por lo tanto se reduce también el costo de los servicios de cuidados intensivos. ${ }^{25.26}$

Algunos hospitales ya cuentan con "laboratorios remotos" en diferentes servicios, mediante los cuales los médicos pueden, a través de monitores activados digitalmente, tener acceso a información demográfica del paciente, a un menú de pruebas analíticas y a un reloj que indica el tiempo que resta para completar el análisis. Aparte del ahorro en costos, la principal ventaja de este sistema es que el microbiólogo del laboratorio central continúa siendo responsable de verificar el resultado final, así como la operación del instrumento analítico. ${ }^{23.27}$

Sin duda alguna podemos afirmar, que el laboratorio clínico continuará realizando los perfiles químicos y las pruebas especializadas ${ }^{28.29}$ Los laboratorios remotos automatizados dan tiempos de

\section{AMC, diciembre 1999, vol 41 (4)}


respuesta muy rápidos, elimina el costo de procesar las muestras, reduce el riesgo de contaminación, reduce la necesidad de entrenamiento especializado y mejora la atención de los pacientes. ${ }^{30}$

Así, vemos como muchas pruebas de laboratorio están retornando a los sitios de atención de los pacientes, donde empezaron en el siglo XIX; nos estamos alejando del paradigma del laboratorio centralizado. ${ }^{31} \mathrm{La}$ descentralización de estos exámenes de laboratorio, constituye un área en crecimiento que provee beneficios a los pacientes y que permite una atención médica más efectiva. Este sistema brinda a los pacientes la posibilidad de un diagnóstico y tratamiento más rápido al contarse con información pronta y segura, esto a su vez repercute en una disminución en el número de consultas. ${ }^{32,33}$

Para evaluar la posible aplicación de estos equipos, cada institución necesita tener información acerca de los instrumentos disponibles, así como conocimiento de las ventajas y desventajas de estas tecnologías, del impacto clínico, del costo del proceso de cambio y de las estrategias para la implementación exitosa.

Los equipos para realizar pruebas "al lado del paciente" son innovaciones tecnológicas que tienen el potencial de mejorar la atención a los pacientes sin aumentar los costos, por lo que pronto se convertirán en parte rutinaria de los servicios de cuidados intensivos y otros. ${ }^{34,35}$

El uso de estos sistemas de pruebas "al lado del paciente", implica realizar pruebas fuera del laboratorio, por lo que deberá ser llevado a cabo por profesionales del área de la salud debidamente adiestrados. El personal encargado debe estar familiarizado con aspectos tales como la precisión, certeza, consideraciones preanalíticas, variación biológica, aseguramiento de la calidad y otros. Estos equipos deben ser sencillos de utilizar y mantener y deben ser rentables, con el fin de que provean información clínica significativa, precisa y segura. ${ }^{32,36}$

Aunque generalmente estas pruebas tienden a ser más costosas, tienen el potencial de disminuir los costos totales, ya que afectan beneficiosamente al paciente, al reducir el tiempo en que se obtienen los resultados. Además, son muy útiles cuando se necesita solamente uno o dos parámetros en vez de una batería completa de pruebas. ${ }^{24}$

Es muy probable que la tendencia a la implementación de biosensores continúe en el próximo siglo, siendo los más aceptados los sensores electroquímicos para cuantificación de electrólitos, glucosa, urea, hematocrito y los oxímetros de pulso. Los sensores implantables de monitoreo continuo serán de gran valor en el caso de las pacientes de las unidades de cuidados intensivos. ${ }^{34,37}$

\section{Análisis de imágenes}

El análisis computarizado de imágenes va a cambiar radicalmente, y en algunos casos va a eliminar el análisis microscópico manual en hematología, inmunohistoquímica, citología, urianálisis y citogenética. ${ }^{33}$

Las herramientas para análisis de imágenes pueden captar, realzar y cuantificar imágenes. Esta tecnología tan útil en la investigación básica se está convirtiendo en una herramienta cada vez más importante en los laboratorios clínicos.

En general, la técnica captura, digitaliza y posteriormente procesa una imagen desde un microscopio, realzando e intensificando algunas áreas y mitigando otras, de manera que el usuario pueda medir, cuantificar, clasificar y analizar los datos. Los sistemas de análisis de imágenes pueden también archivar datos, lo cual es importante en los estudios clínicos. Una vez que se tiene la imagen en el monitor de una computadora, se pueden hacer modificaciones como ajustes de color y la eliminación de lo que no se desea ver. ${ }^{38}$

El sistema de análisis de imágenes consiste en una computadora unida a un procesador de imágenes y a una cámara. La cámara toma una imagen de un campo microscópico y el procesador de imágenes traduce la información analógica en información digital que la computadora puede almacenar. Los programas de la computadora analizan posteriormente los datos. ${ }^{39}$

Las tecnologías computarizadas probablemente revolucionarán el campo de la citología en el próximo siglo. Estas tecnologías serán útiles tanto en el entrenamiento como en la evaluación del personal. ${ }^{40}$

Una aplicación del análisis de imágenes es la morfometría, que es la cuantificación del tamaño, forma y textura de las células y tejidos. Esta técnica permite la evaluación objetiva de características citológicas sutiles que pueden dar información diagnóstica y datos importantes del pronóstico de los pacientes. ${ }^{33}$

El análisis computarizado de imágenes se está utilizando para realizar cariotipos. Este sistema no sólo acelera la evaluación de resultados, sino que también brinda más información que los métodos tradicionales, ya que provee mejor resolución e intensificación de las imágenes. En el pasado, los cariotipos se realizaban manualmente, tiñendo, fotografiando y revelando imágenes de cromosomas, para luego recortarlos y ordenarlos por tamaño para revelar anormalidades. El cariotipo automatizado reemplaza todo esto. El sistema computarizado de análisis de cariotipos elimina el trabajo de fotografía y revelado, facilita el almacenamiento de datos y acelera el proceso.

También se está usando el análisis de imágenes para procesar electroforesis, para la detección y cuantificación en forma muy eficiente, de los productos que se separan y facilitan el análisis estadístico de los datos. ${ }^{41}$ Estos sistemas capturan imágenes de geles de electroforesis y son capaces de presentarlas de muchas formas y prepararlas para ser analizadas por programas de secuenciación de ADN. El análisis de imágenes puede 
correlacionar objetivamente las posiciones de las bandas con tamaños de fragmentos de proteínas o ADN y determinar densidades ópticas para calcular concentraciones.

Conforme disminuyen los costos y aumentan las facilidades para utilizar estos equipos, el análisis de imágenes se convertirá en un instrumento cada vez más importante para muchos laboratorios clínicos.

\section{Robótica}

Los laboratorios deben buscar estrategias para reducir los costos y la robótica podrá disminuir grandemente el requerimiento de personal para labores que son repetitivas, como el transporte y procesamiento de muestras. ${ }^{42,43} \mathrm{Se}$ ha informado que el personal del laboratorio pierde más de $20 \%$ de su tiempo en el transporte de muestras, suministros y documentos. Los robots móviles son un método potencial para liberar a las personas de estas tareas. El uso de estos robots permite al personal del laboratorio dedicarse a las tareas analíticas y administrativas mientras el robot hace las entregas. Esto disminuye los costos y permite que cada resultado sea mejor controlado y revisado por un profesional. ${ }^{25,39,44}$

La gran mayoría de esfuerzos por automatizar el laboratorio se han dirigido a las fases analíticas. Actualmente, la automatización se está enfocando también hacia las fases preanalítica y postanalítica, donde se encuentran los procesos que consumen más tiempo y que son altamente repetitivos. Se ha estimado que las tareas preanalíticas representan el $65 \%$ de todas las labores manuales de un laboratorio. ${ }^{45}$

La tercera generación de sistemas de laboratorio, que apareció alrededor de 1990, automatizó la mayoría de los procedimientos preanalíticos, analíticos y postanalíticos del trabajo de laboratorio, eliminando muchas de las tareas más peligrosas, lentas y tediosas. Estos equipos permiten que el personal se dedique al trabajo de mayor valor como lo es la validación de los resultados o el desarrollo de pruebas en áreas emergentes.

Estos equipos, utilizan sistemas que clasifican y distribuyen los tubos de muestras, separando la muestra en tubos secundarios que el mismo equipo rotula, lo que aumenta la velocidad de procesamiento. Otras aplicaciones incluyen centrifugación automática, eliminación de tapones, carga y descarga automática de los analizadores, repetición automática de pruebas alteradas, validación de resultados e interpretación. El sistema aumenta la productividad y la eficiencia, ya que es capaz de verificar los resultados y de determinar cuáles muestras requieren de repetición. ${ }^{45}$

Muy pronto se podrán procesar muchos tipos de muestras, de principio a fin, sin la intervención humana. Por ejemplo, hay laboratorios de hematología que ya utilizan robots que transportan muestras identificadas con códigos de barras, las cargan y descargan de los analizadores automatizados y realizan los frotis sanguíneos. Estos equipos cuentan con sistemas computarizados que controlan desde el procesamiento de la muestra hasta la interpretación de los resultados. ${ }^{47,48}$

Los rápidos cambios en el área de la salud unidos al avance paralelo de la tecnología han estimulado la evolución de nuevas posibilidades para la automatización del laboratorio. ${ }^{49}$ En Japón, la historia de automatización sistemática en los laboratorios clínicos empezó alrededor de 1981. Actualmente, el 72\% de los hospitales universitarios nacionales de este país utilizan sistemas completamente automatizados. El sistema se basa en tecnología robótica y de transporte automatizado. ${ }^{50}$

A partir de 1990, motivado por la continua disminución en personal especializado, y aprovechando que la economía japonesa se encontraba en auge, se instalaron sistemas automatizados de laboratorio en muchos de los laboratorios clínicos de hospitales nacionales.

A mediados de los 90's, se hizo más evidente la necesidad de sistemas completamente automatizados en otros países alrededor del mundo. Aún antes de 1990 algunos países hicieron intentos de automatización completa, como es el caso de la Clínica Mayo en Rochester, Minnesota, que en 1988 trató de instalar un sistema. Sin embargo, el esfuerzo no tuvo éxito debido a los altos costos y dificultades de servicio del fabricante japonés. Lo mismo ocurrió con otros laboratorios clínicos en Estados Unidos.

Actualmente, en Estados Unidos, existen laboratorios que están completamente automatizados y controlados por robots. Uno de ellos es el laboratorio del Hospital Aultman, en Ohio. Después de pasar varios años planeando y buscando los mejores suplidores de equipo robotizado y computarizado, actualmente son el tercer laboratorio en el país controlado por robots. El objetivo inicial de la automatización era reducir el tiempo de respuesta del servicio de laboratorio, pero después de implantar el sistema, encontraron que los beneficios eran mucho más impresionantes. El tiempo de respuesta de un análisis de rutina se disminuyó de $11 / 2$ hora a 45 minutos. Además, el laboratorio tiene un $40 \%$ más de capacidad que anteriormente. Esto significa que los médicos podrán diagnosticar y tratar a los pacientes más rápido. ${ }^{51}$

En dicho laboratorio, las muestras de sangre, orina, tejidos y otras, identificadas por códigos de barras, llegan al laboratorio por medio tubos neumáticos. El código de barras indica las pruebas que se le realizarán a la muestra. Las muestras se mueven dentro del laboratorio en un transportador y detectores localizados a lo largo de la vía, dan seguimiento a cada muestra y le indican al equipo cuáles pruebas realizar a cada una. Durante las horas pico, el laboratorio puede procesar más de 100 muestras por hora. El laboratorio está diseñado de tal manera que se puedan insertar nuevos equipos en la línea robótica conforme se van desarrollando.

Así como se ha extendido en los últimos años la implementación de sistemas automatizados de laboratorio en Estados Unidos y

10 AMC, diciembre 1999, vol 41 (4) 
Corea, se puede predecir que continuarán diseminándose por todo el mundo hasta llegar a ser instalados en la mayoría de laboratorios para el año $2010 .^{51}$

Los sistemas robóticos diseñados específicamente para automa-

- tizar las tareas del laboratorio ya se encuentran disponibles comercialmente. Equipados con computadoras, programas analíticos y de soporte, estos equipos están revolucionando el concepto de laboratorio clínico. Sin embargo, quedan todavía muchos desafíos que superar relacionados al entrenamiento de los individuos que desarrollan y manejan los laboratorios robóticos y al desarrollo rentable de esta nueva tecnología. ${ }^{52}$ En muchos países del mundo, la automatización sistematizada de los laboratorios no ha sido posible debido a que la tecnología actual es demasiado costosa y no está suficientemente estandarizada para satisfacer las necesidades de diferentes mercados.

En el laboratorio clínico será cada vez más común el uso de robots. La mayoría serán utilizados para reducir tareas monótonas como la preparación de las muestras, para minimizar la exposición humana a ambientes peligrosos o para llevar a cabo grandes cantidades de procedimientos experimentales repetitivos, con lo cual se obtendrán resultados más precisos y exactos. Ejemplos típicos de la implementación de robots en el laboratorio clínico son el transporte de muestras, la automatización de la preparación de la muestra, separación y alicuoteo. ${ }^{53,54}$

Utilizando robots versátiles se pueden automatizar algunas de las tareas que consumen más tiempo asociadas a la reacción en cadena de la polimerasa, como el dispensar de los reactivos. ${ }^{55}$

Los sistemas robotizados reducen el trabajo y el tiempo en que se producen los resultados de muestras críticas, lo que ayuda al médico a tomar decisiones relevantes en el tratamiento de pacientes en estado delicado. ${ }^{26,56}$ Se están desarrollando instrumentos más independientes, con sistemas de computación capaces de determinar características de la muestra como cantidad insuficiente, código de barras no legible, muestras de pobre calidad, recipiente inadecuado o muestras dañadas. En el futuro se diseñarán equipos que sean capaces de funcionar por meses sin necesidad de mantenimiento preventivo, suministro de reactivos o reparaciones. ${ }^{26}$

\section{Sistemas de manejo de información}

Entre los factores que definen el laboratorio del futuro están el desarrollo de sistemas de comunicación computarizados, inteligencia artificial, sistemas robóticos y sistemas de almacenamiento y recuperación de datos. ${ }^{57} \mathrm{El}$ laboratorio es la mayor fuente de datos para el diagnóstico de las enfermedades de los pacientes. Tiene una importantísima, aunque no muy reconocida, función en la atención médica como fuente, moderador y conductor de información vital para la detección, confirmación y registro de enfermedades. La información del laboratorio debe estar integrada a la información clínica y diagnóstica de cada paciente, para tener una base sobre la cual evaluar el manejo que se les da. Se necesitan sistemas integrados con los que el laboratorio clínico pueda controlar la producción de datos desde muchos sitios y por medio de los cuales pueda recolectar, dirigir y almacenar resultados de cada paciente. ${ }^{58,59}$

La tendencia actual en las políticas de salud sugiere que las bases de datos de los pacientes deben tener un alcance global, es decir que no se limiten a un solo laboratorio u hospital. La estructura de los sistemas modernos de información clínica enlaza redes regionales y nacionales con redes de área local que unen los diferentes componentes del sistema de salud.

Se están desarrollando sistemas de información y manejo de imágenes que consolidan lo que actualmente son sistemas separados, en una solución totalmente integrada. El sistema permitirá accesar eficientemente la información del laboratorio desde cualquier punto del área hospitalaria. El resultado será un tiempo de informe de resultados mucho menor, un acceso rápido al historial del paciente (reportes e imágenes) y sobre todo un aumento en la satisfacción del paciente y del médico por el servicio dado. ${ }^{18,60}$

También, se han ideado sistemas portátiles que le permiten al médico solicitar convenientemente exámenes de laboratorio y recibir los resultados, reduciendo tanto el costo como el tiempo en que el laboratorio produce los resultados de exámenes. Los sistemas de reconocimiento de la voz van a obviar la necesidad de personas que transcriban la información.

En los años 80's, muchos hospitales comenzaron a utilizar sistemas médicos asistidos por computadoras y el laboratorio clínico empezó a adaptarse al cambio, desarrollando sistemas de procesamiento de información. Los laboratorios clínicos se han visto forzados a transformarse de un servicio de procesamiento de muestras a centros de procesamiento de información. El desarrollo de sistemas de información en el laboratorio puede cambiar la forma en que se brinda atención médica. El laboratorio debe estar diseñado de manera que los datos acumulados puedan ser utilizados efectivamente para investigación y para la atención al paciente.

Cuando el laboratorio está respaldado por un sistema de información hospitalaria, la recolecta de las muestras puede estar asistida por información sobre el volumen necesario, cómo y en qué tipo de recipiente se debe obtener la muestra, etc. Cuando se cuenta con sistemas de este tipo, se involucra a todo el personal médico, debido a que la solicitud de exámenes y el etiquetado de muestras es llevado a cabo en el mismo sitio de recolección de la muestra.

Las muestras son transportadas directamente a los analizadores, los cuales son capaces de leer automáticamente las etiquetas de códigos de barras. Esto indica que los análisis se realizan sin necesidad de papeles, lo que ahorra mucho tiempo. 
En el pasado era imposible intercambiar información entre diferentes departamentos de un hospital. Sin embargo, con las computadoras, toda la información médica de cada paciente es almacenada y los datos necesarios pueden obtenerse en instantes. Como resultado, desde el punto de vista del laboratorio, toda la información necesaria para el procesamiento de muestras se puede obtener del expediente de cada paciente. A la vez, el laboratorio debe contar con sistemas por medio de los cuales se puedan incorporar todos los resultados (datos numéricos, análogos, ópticos, electrónicos y texto) al expediente de cada paciente, para que tenga libre acceso para otros departamentos a cualquier hora del día y desde cualquier terminal. ${ }^{61}$

La ciencia busca de sistemas de información que aumenten la eficiencia, la competitividad y que resulte en un incremento de la satisfacción de los pacientes, producto de una atención rápida y de alta calidad. ${ }^{55}$ El propósito de cualquier sistema computarizado de manejo de información es que sea capaz de recopilar, integrar, comparar, analizar y distribuir la información interna y externa de la organización, en forma oportuna, efectiva y eficiente, con la cual se tienen las herramientas necesarias para la toma de decisiones.

En el futuro, la telemedicina y telepatología permitirán que cualquier laboratorio pueda unirse con expertos de centros especializados para realizar consultas. La telepatología consiste en utilizar tecnologías de telecomunicación (Internet, líneas de fibra óptica y líneas telefónicas) para transmitir datos patológicos e imágenes entre dos o más sitios remotos con fines diagnósticos, educativos y de investigación. El sistema consiste en una cámara de video de alta resolución incorporada a un microscopio, la cual toma imágenes que son transmitidas a un sitio remoto.

Las consultas de telepatología pueden ser transmitidas usando imágenes estáticas o sistemas dinámicos mediante el cual se transmiten imágenes en vivo. Este último sistema, le permite al receptor controlar y manipular las imágenes, jugando un papel activo en la selección de los mejores campos diagnósticos y ajustando el foco y la iluminación. ${ }^{62}$

La telepatología promete ser un instrumento valioso para el laboratorio clínico ya que se pueden hacer consultas de láminas y otros materiales desde sitios remotos para obtener segundas opiniones de casos difíciles por parte de expertos en el campo, en forma sumamente rápida y económica. Esto es muy útil especialmente para los laboratorios de hospitales rurales, que generalmente carecen de personal especializado, lo que limita los servicios que puede ofrecer. ${ }^{63}$

Además de ser clínicamente deseable, la telepatología es tecnológicamente factible. La red Internet parece ser un medio prometedor de bajo costo, por medio del cual se intercambien consultas internacionales e interinstitucionales.

\section{AMC, diciembre 1999, vol 41 (4)}

La claridad de las imágenes de video que se obtienen actualmente, permite que la telepatología pueda también ser utilizada en la supervisión remota de los programas de aseguramiento de la calidad en laboratorios de microbiología, así como para el entrenamiento a distancia del personal. ${ }^{63}$

Todas estas nuevas tecnologías serán costosas de implementar, pero si se planean bien se puede llegar a disminuir los costos y a mejorar la calidad de la atención médica a los pacientes. ${ }^{64} \mathrm{El}$ despliegue de la nueva tecnología de información mejorará la calidad y la eficiencia de las operaciones de los laboratorios. Los cambios que están ocurriendo son muy grandes y exigen que el papel del profesional médico y de laboratorio se modifique en respuesta al nuevo ambiente de información. ${ }^{65}$

\section{Situación en Costa Rica}

En los hospitales de Costa Rica, públicos y privados, apenas estamos comenzando a ver la introducción de algunas de las tecnologías mencionadas anteriormente. En particular, las más utilizadas son las pruebas rápidas que se realizan "al lado del paciente". También, en algunos laboratorios de hospitales se están haciendo esfuerzos para comenzar a utilizar, en la práctica clínica, las técnicas de diagnóstico molecular por medio de PCR. Por otro lado, se están haciendo esfuerzos por mejorar los sistemas de manejo de información con que cuentan actualmente los laboratorios.

Igual que ocurre en otros países, en el presente, aún los analizadores automáticos más grandes, se están volviendo obsoletos en muchos laboratorios de nuestro país, donde se debe entregar información flexible que se acople a las necesidades siempre cambiantes del laboratorio clínico y del sistema de salud.

Las presiones administrativas y económicas para reducir costos pero aumentando la productividad, son desafíos que van a estimular la introducción de tecnologías modernas como las discutidas anteriormente. Solo de esta manera, pueden los laboratorios clínicos de este país contribuir a mejorar la calidad de la atención médica de todo los hospitales. No cabe duda de que los laboratorios de nuestro país deben actualizarse en este ámbito tecnológico, para que puedan cumplir con sus objetivos y metas.

\section{Conclusiones}

Claramente, lo mejor está por venir. Conforme progresa la tecnología, el laboratorio será más eficiente y más libre de errores y será capaz de satisfacer las crecientes y cambiantes necesidades de los pacientes y de los médicos. El microbiólogo y el laboratorio tendrán cada vez un mayor impacto en la calidad de atención de los pacientes. La mayoría de avances tecnológicos en el laboratorio clínico están dirigidos por el objetivo fundamental de la optimización del servicio brindado. 
El laboratorio clínico está en un estado de cambio. Las instituciones médicas están afrontando los retos contradictorios de mejorar y aún expandir los servicios de laboratorio, pero a la vez reduciendo los gastos generales de operación. En esta necesidad de "hacer más con menos", los directores de laboratorios deben encontrar nuevas formas para reducir los gastos en un laboratorio que de antemano ha estado operando con presupuestos sustancialmente reducidos. La tecnología asumirá un papel primordial en la necesaria reestructuración de los laboratorios clínicos y podrá ser la llave para lograr esta meta. ${ }^{26,28}$

Conforme surgen nuevas tecnologías, así aumentan las oportunidades de los profesionales de laboratorio de participar activamente en equipos dedicados al proceso de seleccionar las pruebas, instrumentos y metodologías que brinden los resultados óptimos y que cumplan con los criterios más estrictos de calidad, certeza, precisión y tiempo de respuesta. Estos equipos de trabajo serán los responsables de determinar cómo, cuándo y dónde se deben implementar pruebas fuera del laboratorio clínico central, así como de la interpretación y apropiada utilización de los resultados. No podemos perder de vista que todas estas máquinas e instrumentos novedosos deben ser controladas por personas preparadas. Para poder obtener de esta nueva tecnología una eficiencia óptima y ahorro en costos, se requerirá la participación y cooperación de profesional médico y de laboratorio, quienes deberán adiestrarse en computación, electrónica, sistemas de ingeniería y robótica, para poder estar a la altura de la nueva tecnología que nos llega. ${ }^{66,67}$

Para implementar nueva tecnología en nuestros laboratorios, se deben tomar en cuenta una serie de factores internos y externos. Dentro de los internos se tienen que considerar las limitaciones de recursos financieros, materiales, humanos y de infraestructura con que cuentan las organizaciones prestadoras del servicio de salud. Los externos son las necesidades de los usuarios, decisiones políticas, localización geográfica del servicio, vías de comunicación, etc. Otros factores que demandan consideración son la exactitud, el ahorro de tiempo previsto, el costo de los reactivos e instrumentos relacionados y el potencial para diversas aplicaciones. ${ }^{68}$

Cuando se habla de recursos financieros se requiere hacer estudios de factibilidad y rentabilidad, del costo de un nuevo sistema, unido al rendimiento que se puede obtener en cada caso. Otro factor a tomar en cuenta es el beneficio para el individuo, la familia y la comunidad, evaluando el área geográfica en donde se encuentra ubicado el laboratorio.

\section{Abstract}

In this paper, some of the technologies which will have the greatest impact on clinical laboratory techniques of the future are discussed. These include: molecular diagnostics, point-ofcare testing, computerized image analysis, robotics and information management systems. A review of the main applications and advantages that these new technological advances will bring to the performance of the clinical laboratory in the twenty-first century, is also presented.

Key words: technology, molecular diagnostics, near patient testing, point-of-care testing, biosensors, robotics, image analysis, information management, telepathology, clinical laboratory.

\section{Referencias}

1. Smith BL, Vender JS. Point-of-care testing. Respir Care Clin NAm 1995; $1: 133-141$.

2. Staneck L. Impact of technological developments and organizational strategies on clinical laboratory cost reduction. Diagn Microbiol Infect Dis 1995; 23: 61-73.

3. Guder WG, Buttner J. Clinical chemistry in laboratory medicine in Europe-past, present and future challenges. Eur J Chem Clin Biochem 1997; 35: 487-494.

4. Burtis CA. Advanced technology and its impact on the clinical laboratory. Clin Chem 1987; 33: 352-357.

5. Mulcahy GM. The integration of molecular diagnostic methods into the clinical laboratory. Ann Clin Lab Sci 1999; 29; 43-54.

6. Kiechle FL, Zhang X, Malinski T. The molcular pathology laboratory of the 21st century. Ann Clin Lab Sci 1999; 29: 59-77.

7. De Cresce RP, Lifshitz MS. PCR (polymerase chain reaction) and the future of molecular testing. MLO Med Lab Obs 1993; 25: 28-33.

8. Gottfredsson M. Cox GM, Perfect JR. Molecular methods for epidemiological and diagnostic studies of fungal infections. Pathology 1998; 30: 405-418.

9. Hall GS. Probe technology for the clinical microbiology laboratory. Arch Pathol Lab Med 1993; 117: 578-583.

10. Tsongalis GJ, Coleman WB. Molecular oncology: diagnostic and prognostic assessment of human cancers in the clinical laboratory. Cancer Invest 1998; 16: 485-502.

11. Zinder O. New directions in laboratory-clinician communications. Clin Chim Acta 1998; 278: 83-94.

12. Maron BJ, Moller JH, Seidman CE, Vincent GM, Dietz HC, Moss $\mathrm{AJ}$, et al. Impact of laboratory molecular diagnosis on contemporary diagnostic criteria for genetically transmitted cardiovascular diseases: hypertrophic cardiomyopathy, long-QT syndrome, and Marfan syndrome. Circulation 1998; 98: 1460-1471.

13. Niemeyer DM. Polymerase chain reaction: a link to the future. Mil Med 1998; 163: 226-228.

14. Ross JS. Molecular pathology and managed care: challenges and opportunities. Diagn Mol Pathol 1998; 7: 189-191. 
15. Zaloga GP, Hill TR, Strickland RA, Kennedy D, Visser M, Ford K, et al. Bedside blood gas and electrolyte monitoring in critical ill patients. Crit Care Med 1989; 17: 920-925.

16. Kost GJ. New whole blood analyzers and their impact on cardiac and critical care. Crit Rev Lab Sci 1993; 30: 153-20.

17. Hobbs FD, Delaney BC, Fitzmaurice DA, Wilson S, Hyde CJ, Thorpe $\mathrm{GH}$, et al. A review of near patient testing in primary care Health Technol Assess 1997; 1: 1-229.

18. ADAC Healthcare Information Systems. Future Technology Today. Image Management Solutions.

19. Dirks JL. Diagnostic blood analysis usung point-of-care technology. AACN Clin Issues 1996; 7: 249-259.

20. Hunter KW Jr. Biosensors. A new analytic technology for realtime, on-line biochemical monitoring. A J Clin Pathol 1989; 91: 32-33.

21. Gizeli E, Lowe CR. Immunosernsors. Curr Opin Biotechnol 1996; 7: 66-71.

22. Pace SJ. Biosensors and the clinical laboratory. Med Instrum 1985; 19: $168-172$.

23. Felder R. Robotics and automated worksations for rapid response testing, Am J Clin Pathol 1995; 104: 26-32.

24. Harcey MA. Point-of-care laboratory testing in critical care. Am J Critl Care 1999; 8: 72-83.

25. Felder RA, Savory J, Margrey KS, Holman JW, Boyd JC. Development of a robotic near patient testing laboratory. Arch Pathol Lab Med 1995; 119: 948-951.

26. Felder RA. Robotics and automated workstations for rapid response testing . Am J Clin Pathol 1995; 104: 26-32.

27. Kost GJ, Hague C. The current and future status of critical care testing and patient monitoring. Am J Clin Pathol 1995; 104: 2-17.

28. O'Kane DJ, Ebert TA, Hallaway BJ, Roberts SG, Bhuiyan AK, Tenner KS. A laboratorian's perspective on evaluation and implementation of new laboratory tests. Clin Chem 1997; 43: 1771-1780.

29. Belsey R, Baer D, Sedwell D. Laboratory test analysis near the patient. Opportunities for improved clinical diagnosis and management. JAMA 1986; 255: 775-786.

30. Felder RA, Savory J, Margrey KS, Holman JW, Boyd JC. Development of a robotic near patient testing laboratory. Arch Pathol Lab Med 1995; 119: 948-951.

31. Friedman BA. The challenge of managin laboratory information in a managed care environment. Am J Clin Pathol 1996; 105: 3-9.

32. Jacobs E, Laudin AG. The satellite laboratory and point-of-care testing. Integration of information. A J Clin Pathol 1995; 104: 33-39.

\section{AMC, diciembre 1999, vol 41 (4)}

33. Erler BS, Chein K, Marchevsky AM. An image analysis worksation for the pathology laboratory. Mod Pathol 1993; 6: 612-618.

34. Harvey MA. Point-of-care laboratory testing in critical care. Am J Crit Care 1999; 8: 72-83.

35. Castro HJ, Oropello JM, Halpern N. Point-of-care testing in the intensive care unit. The intensive care physician's perspective. Am J Clin Pathol 1995; 104: 95-99.

36. Smith I. Revolution in varying degrees. Ins Med Lab Sci 1993; 277-280.

37. Woo J, Henry JB. The advance of technology as a prelude to the laboratory of the twenty-first century. Clin Lab Med 1994; 14: 459-471.

38. Cardullo, RA. Introduction to Image Processing. Methods in Cell Biology. 1997; 56: 91-115.

39. Lewis R. Applications Of Image Analysis Systems Expand Beyond The Research Lab. The Scientist 1996; 10:18-19.

40. Vooijs GP, Davey DD, Somrak TM, Goodell RM, Grohs DH, Knesel EA, et al. Computarized training and proficiency testing. International Academy of Cytology Task Force summary. Diagnostic Cytology Towards the 21st Century: An International Expert Conference and Tutorial. Acta Cytol 1998; 42: 141-147.

41. Appel RD, Palagy PM, Walther D, Vargas JR, Sanchez JC, Ravier F, et al. Melanie II -a third-generation sofware package for analysis of two-dimensional electrophoresis images: I. Features and user interface. Electrophoresis 1997; 18: 2724-2734.

42. Boyd JC, Felder RA, Savory J. Robotics and the changing face of the clinical laboratory. Clin Chem 1996; 42: 1901-1910.

43. Place JF, Truchaud A, Ozawa K, Pardue H, Schnipelsky P. Use of artificial intelligence in analytical systems for the clinical laboratory. Clin Biochem 1995; 28: 373-389.

44. Wood MD, Franchetti JA. Laboratory automation using robotics and information management systems. Curr Opin Biotechnol 1993; 4: 91-94

45. McPherson RA. Robotics, automation, and the new role of process control. Clin Lab Manage Rev 1998; 12: 339-346.

46. Hoffmann GE. Concepts form the third generation of laboratory systems. Clin Chim Acta 1998; 278: 203-216.

47. Diamond LW, Nguyen DT, Sheridan BL, Strul M, Bailey K, Bak A. An automated hematology laboratory with computer-controlled robotics. Medinfo 1995; 8: 620-623.

48. Rothe M Wingfield S, Barranco P, Charache S. Robotics in the hematology laboratory. An evaluation of the productivity of the Sysmex HS-330. Am J Clin Pathol 1995; 103: 154-158.

49. Boyd JC, Felder RA, Savory J. Robotics and the changing face of the clinical laboratory. Clin Chem 1996; 42: 1901-1910. 
50. Sasaki M, Kageoka T, Ogura K, Kataoka H, Ueta T, Sugihara S. Total laboratory automation in Japan. Past, present, and the future. Clin Chim Acta, 1998; 278: 217-227.

51. Felder RA. Robotics and automated worksations form rapid response testing. Am J Clin Pathol 1995; 104: 26-32.

52. Felder RA, Boyd JC, Margrey K, Holman W, Savory J. Robotics in the medical laboratory. Clin Chem 1999; 36: 1534-1543.

53. Ozawa K, Schnipeslky P, Pardue HL, Place J, Truchaud A. International Federation of Clinical Chemistry. The expanding role of robotics in the clinical laboratory. Ann Biol Clin 1999; 59: 528535 .

54. Felder RA. Laboratory systems integration: robotics and automation. Ann Biol Clin 1991; 49: 298-300.

55. Turner R, Felder RA, Kealy M. Automation of the polymerase chain reaction. Biotechnol Lab 1995; 13:50-51.

56. Estey C, Nagy D, Onaghise J, Herold D, Felder R. A Multi-center study examining the implementation of mobile robots in a clinical laboratory. Mobile Robots in the Clinical Laboratory. California Computer Research Inc.

57. Beugelsdijk TJ. The future of laboratory automation. Genet Anal Tech Appl 1991; 8: 217-220.

58. Weilert M. The clinical laboratory is in the information business. Clin Chem Acta 1994; 224: 1-7.

59. McDowall RD. An apdate on laboraory information managenemt systems. Journal Pharm Biomed Anal 1993; 11: 1327-1330.
60. Buffone GJ, Moreau DR. Laboratory computing -process and information management supporting high- quality, cost-effective healtcare. Clin Chem 1995; 41: 1338-1344.

61. Leonard DG. The future of molecular genetic testing. Clin Chem 1999; 45: 726-731.

62. Ivan A. Telepathology: Is There a Future? Telemed Today 1996; 4: 24-26.

63. McLaughlin WJ, Schifman RB, Ryan KJ, Manriquez GM, Bhattacharyya AK, Dunn BE, Weinstein RS. Telemicrobiology: feasibility study. Telemed J 1998; 4: 11-17.

64. Kasahara Y, Ashihara Y. Simple devices and their possible application in clinical laboratory downsizing. Clin Chem Acta 1997; 267: 87-102.

65. Friedman BA, Mitchell W. The deployment of information technology in clinical laboratories and its impact on professional roles. Clin Lab Manage Rev 1992; 6: 87-93.

66. Woo J, henry JB. The advance of technology as a prelude to the laboratory of the twenty-first century. Clin Lab Med 1994; 14: $59-71$.

67. Scott NCH. Desktop laboratory testing in laboratory practice. Brithish Med J 1989; 299: 579-580.

68. Jenkins SG. Evaluation of new technology in the clinical microbiologhy laboratory. Diagn Microbiol Infect Dis 1995; 23: 53-60. 